University of Louisville

ThinkIR: The University of Louisville's Institutional Repository

\title{
Effects of short term high fat diet on obesity and perivascular adipose structure and function in mice : role of aldose reductase, and aldehyde metabolizing enzyme.
}

Daniel Alan Murphy

University of Louisville

Follow this and additional works at: https://ir.library.louisville.edu/honors

Part of the Medical Sciences Commons

\section{Recommended Citation}

Murphy, Daniel Alan, "Effects of short term high fat diet on obesity and perivascular adipose structure and function in mice : role of aldose reductase, and aldehyde metabolizing enzyme." (2013). College of Arts \& Sciences Senior Honors Theses. Paper 18.

http://doi.org/10.18297/honors/18

This Senior Honors Thesis is brought to you for free and open access by the College of Arts \& Sciences at ThinkIR: The University of Louisville's Institutional Repository. It has been accepted for inclusion in College of Arts \& Sciences Senior Honors Theses by an authorized administrator of ThinkIR: The University of Louisville's Institutional Repository. This title appears here courtesy of the author, who has retained all other copyrights. For more information, please contact thinkir@louisville.edu. 
Effects of Short Term High Fat Diet on Obesity and Perivascular Adipose Structure and Function in Mice:

Role of Aldose Reductase, an Aldehyde Metabolizing Enzyme

By

Daniel Alan Murphy

Submitted in partial fulfillment of the requirements

for Graduation (summa or magna) cum laude

and

for Graduation with Honors from the Department of Biology (if pertinent)

University of Louisville

May, 2013 


\section{Table of Contents}

Abstract:....................................................................................................................... 3

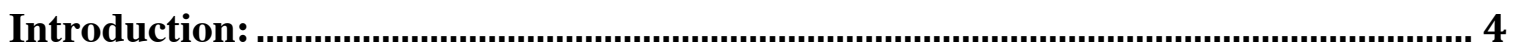

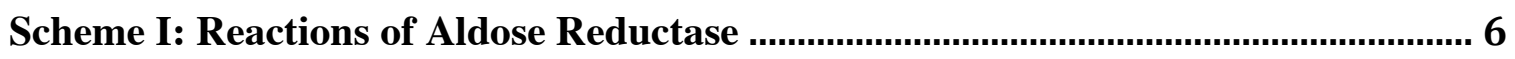

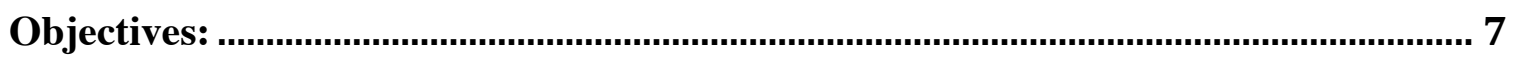

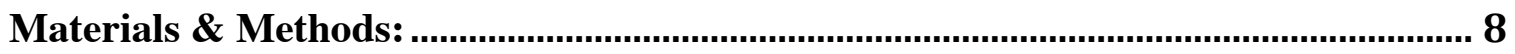

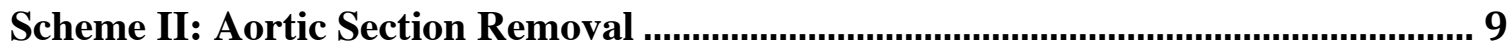

Scheme III: Dorsal and Ventral Adipose Defined ..................................................... 10

Figure 1: White Adipocyte Clusters..................................................................... 11

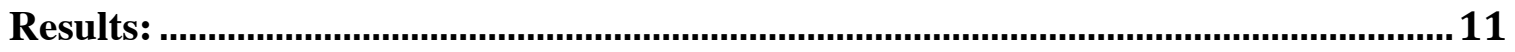

Figure 2: Total Body Fat and PVAT Ring Analysis ............................................ 16

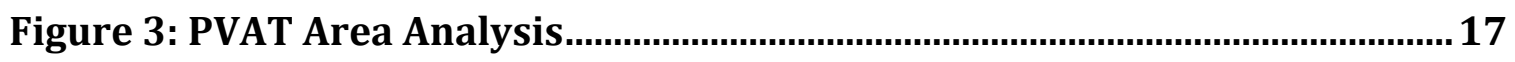

Figure 4:White Adipocyte Cluster Analysis ............................................................ 18

Figure 5: White Adipocyte Area Analysis.................................................................. 19

Figure 6: Cluster Adipocyte Enumeration ............................................................. 20

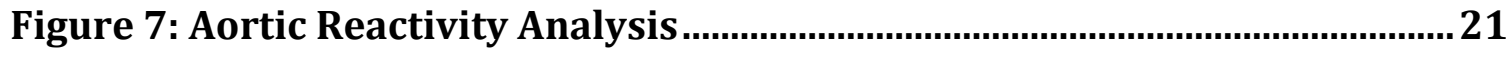

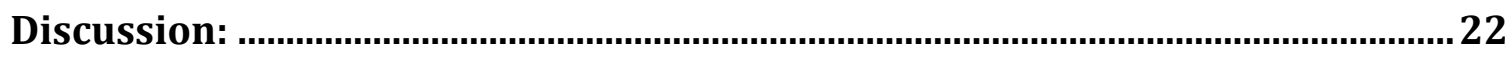

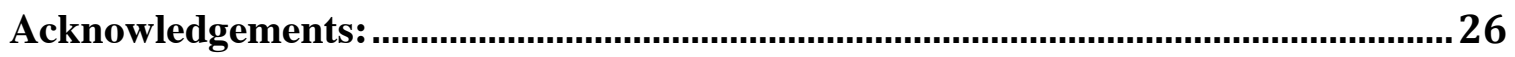

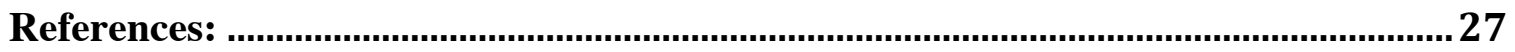




\begin{abstract}
:
This study was undertaken to determine the effects of high fat diet and aldose reductase deficiency on the structure and function of murine perivascular adipose tissue. Wild type (WT) (C57 bl/6) and aldose reductase knockout (AR-null) mice were aged to 8 or 18 weeks on a normal chow diet then some were switched to a high fat diet for 4 weeks while others remained on the control diet for the same amount of time. Following 4 weeks, mice were euthanized PVAT was analyzed. High fat feeding significantly increased body fat percentage in both age groups of mice and the area of PVAT present around distal thoracic aorta sections also increased. Additionally, WT and AR-null mice appeared to exhibit an increase in the area of white adipocyte clusters present in PVAT, although the results were not significant. When examining the area of white adipocytes present in PVAT, HF feeding significantly increased area of adipocytes throughout PVAT in WT, 22-week old mice and a significant increase in VAT and overall PVAT, but not DAT in AR-null mice. To examine the functional properties of PVAT, sections of aorta with intact and removed PVAT were measured for contraction and relaxation properties. In WT mice, HF feeding resulted in endothelial dysfunction, seen as a reduced relaxation response to acetylcholine. The presence of intact PVAT reversed the observed dysfunction. Collectively, these data suggest that PVAT adiposity is increased similar to overall adiposity with high fat feeding and that this change is structure may contribute to the functional changes also observed with high fat feeding.
\end{abstract}




\section{Introduction:}

Obesity is one of the fastest growing health problems today, nearing pandemic status worldwide. The United States is perhaps one of the countries most affected, with roughly one third of the population being obese [2]. A diet composed of high fat and sugar laden foods and beverages, along with an ever more sedentary lifestyle, has spelled a recipe for disaster in American citizens. Obesity is particularly prevalent in the Deep South, where Mississippi and Louisiana have obesity rates of $34.9 \%$ and $33.4 \%$, respectively [3]. With an obesity prevalence of $30.4 \%$, Kentucky is also no stranger to this "growing" problem [3].

Obesity is associated with an increased risk of developing type-2 diabetes and cardiovascular disease. This increased cardiovascular risk is associated with increased visceral adiposity, but not subcutaneous adiposity [4]. However, the mechanisms by which visceral adiposity affects cardiovascular disease risk are currently unknown. In recent years, diabetes has also become an increasing problem in the United States. Type2 diabetes occurs when the body either does not respond to insulin, or no longer produces enough insulin to elicit a response in the body [5]. It is proposed that the release of free fatty acids from adipocytes blocks insulin-signaling pathways, leading to insulin resistance [5].

No direct link between visceral adiposity and cardiovascular disease has been elucidated to date. Because of this, investigations of more local adipose depots has ensued and the perivascular adipose tissue (PVAT) has become an increasingly popular topic in cardiovascular research. Found surrounding the adventitial layer of blood vessels, perivascular adipose exhibits a reduced level of adipocyte differentiation compared to visceral and subcutaneous adipose depots [6]. In addition, perivascular adipocytes 
possess peculiar properties such as endocrine functions [7], as well as reactivity to metabolic cues and functioning in signal transduction to blood vessels [8-10]. PVAT exhibits anti-contractile effects on blood vessels via the release of vasorelaxants such as adiponectin and yet undiscovered adipose derived relaxing factors (ARDF) [11]. Under normal physiological conditions, PVAT protects against neointimal hyperplasia following angioplasty, potentially a result of ADRF and adiponectin secretions [12].

Perivascular adipose is believed to be a key contributor to cardiovascular disease, particularly atherosclerosis. Magnetic resonance imaging (MRI) studies show that the quantity of PVAT on human coronary arteries is correlated with hyperglycemia, hypertriglyceridemia, and coronary atherosclerosis [13]. Additionally, studies where perivascular adipose has been transplanted to vessels where it is normally absent show that PVAT contributes to neointimal hyperplasia following intraluminal vascular injury [14]. It is possible that these deleterious contributions of PVAT are a result of increased pro-inflammatory cytokine secretion under conditions of obesity [15].

Aldose reductase (AR), a widely expressed aldehyde-metabolizing enzyme, functions in the reduction of cytotoxic aldehydes such as those formed during lipid peroxidation [1]. Glucose acts as an incidental substrate to AR because of a very high $\mathrm{Km}$ [1], yet AR-catalyzed metabolism of glucose via the polyol pathway is associated with secondary diabetic complications, potentially due to accumulation of sorbitol (Scheme I) [16]. In previous years, inhibition of the AR enzyme has been considered as a possible therapeutic target for managing type-1 diabetes [17]. Inhibition of AR in type-1 diabetic models shows favorable effects on vascular diabetic complications such as retinopathy, neuropathy, nephropathy, and endothelial dysfunction [17]. In type-2 diabetes, however, 
the role of AR in presently unclear because blood glucose does not reach the levels observed in Type I diabetes models of human disease.

\section{Scheme I: Reactions of Aldose Reductase}

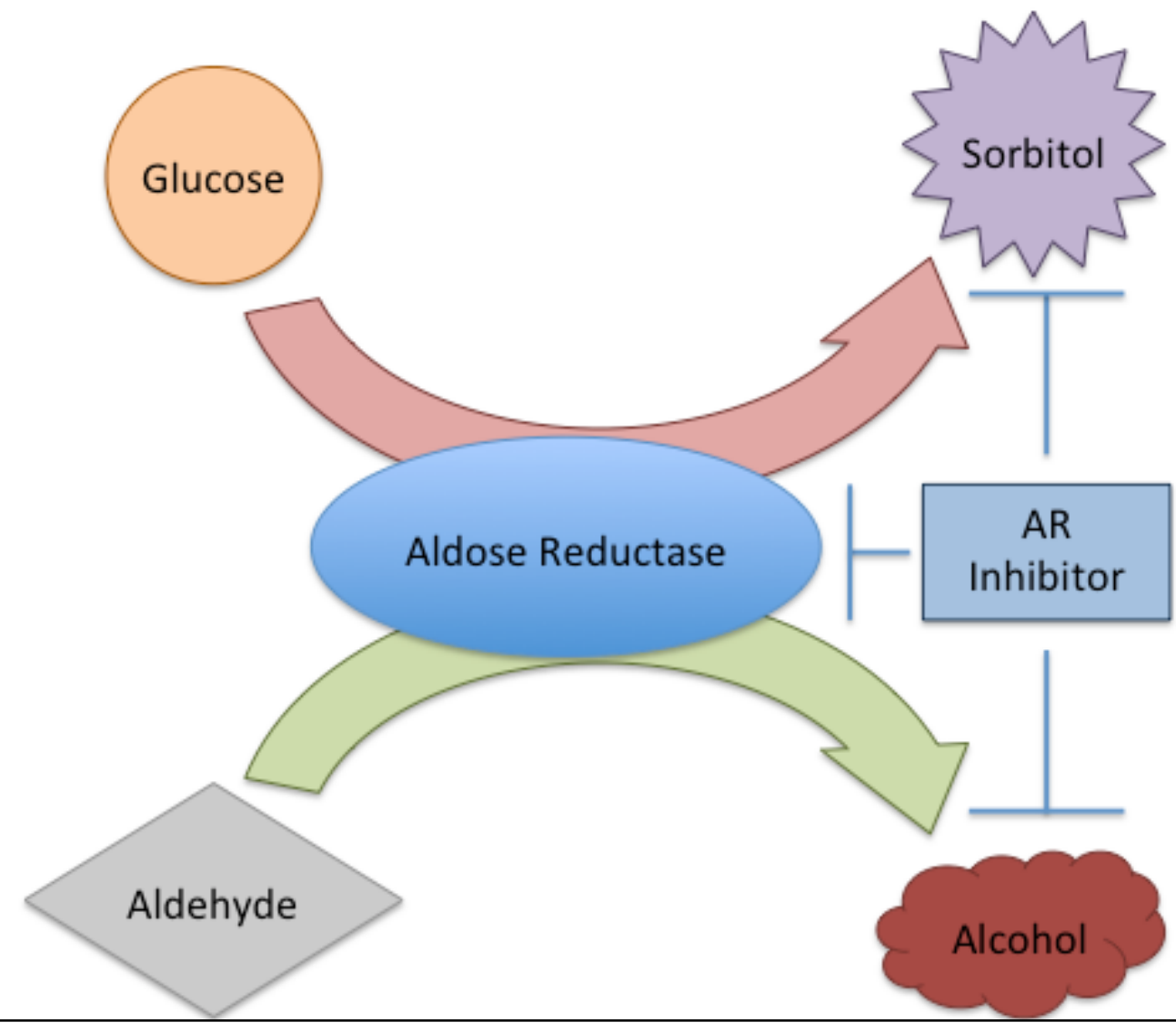

Scheme I: Illustration of aldose reductase function of reducing aldehydes to alcohols. The enzyme's affinity for glucose is relatively low [1], and at normal blood sugar levels sorbitol production is minimal. In a type-1 diabetic state, where blood sugar levels are very high, sorbitol production is much higher. Generation of sorbitol, which is an osmolyte, to high intracellular levels could increase cell swelling and impair function.

The objective of this study was to provide an in depth characterization of aortic perivascular adipose tissue in control diet (CD) and high fat (HF) diet fed mice. Second, we wanted to investigate the potential role of aldose reductase (AR) in modifying the response to HF diet because AR deficiency should diminish the ability of the mouse to deal with enhanced levels of oxidative stress via the reduction of cytoxic aldehydes to 
less reactive alcohols. It has been shown that high fat diet induces endothelial dysfunction [19], and perivascular adipose may play an important role in this process [11], although exact mechanisms are not known. The goal of this study is to gain a better understanding of how diet affects obesity and PVAT structure and function because PVAT may ultimately contribute to vascular dysfunction and disease [20]. Studies of PVAT have revolved primarily around white unilocular adipocytes present in this depot that are shown to increase risk for atherosclerosis and cardiovascular disease [13-15]. Murine thoracic aorta PVAT is primarily brown multilocular adipose under normal conditions, and it is considered resistant to inflammation induced by obesity [21]. To test this idea, we hypothesized that high fat diet feeding will result in an increased PVAT area, as well as a shift in PVAT composition from a brown adipose phenotype to a white adipose phenotype. Recent evidence suggests that an accumulation of oxidative stress in or around the vasculature under obese conditions results in endothelial dysfunction, diabetes, and an elevated cardiovascular disease risk [22]. Because AR can convert cytotoxic aldehydes to alcohols [1], and thus, decrease products of oxidative stress, we hypothesized that mice genetically deficient of AR (AR-null mice [23]) would be more susceptible to diet-induced obesity, exhibiting increased adiposity and vascular functional complications associated with obese conditions.

\section{Objectives:}

1) To determine if PVAT adiposity is increased under high fat diet feeding conditions, as is overall adiposity;

2) To examine the effects of high fat diet on functional properties of PVAT in isolated aorta; and,

3) To assess whether genetic deletion of AR affects obesity and PVAT structure and function under control and high fat diet feeding conditions. 


\section{Materials \& Methods:}

Mice. For this study, young male wild type (WT) (C57B1/6J; Jackson Laboratory, Bar Harbor, MA) and aldose reductase-null (AR-null) mice bred on a C57Bl/6 background [23] were maintained in a temperature-controlled room $\left(22^{\circ} \mathrm{C}\right)$ on a $12: 12 \mathrm{hr}$ light: dark cycle and fed a normal chow (NC) diet composed of 59\% kcal from carbohydrates, $29 \%$ kcal from protein, and 12\% kcal from fat (LabDiet 5010, PMI Nutrition, St. Louis, MO) after weaning. At 8- or 18-weeks old, mice were either maintained on NC diet or switched to a high fat (HF) diet composed of $60 \% \mathrm{kcal}$ from fat (lard-based), $20 \% \mathrm{kcal}$ from protein, and 20\% kcal from carbohydrates (D12492, Research Diets INC, New Brunswick, NJ) for 4 weeks.

Fat Composition: Body lean and fat masses were analyzed and their percentages calculated using dual energy x-ray absorptiometry (DEXA) scans using a Lunar PIXImus (GE Healthcare, Waukesha, WI) bone density scanner following sodium pentobarbital injection (0.15-0.3 mL, Sigma-Aldrich, St. Louis, MO). The composition of mouse head (majority bone) was excluded from all measurements (Fig. 1A).

Aorta Reactivity: Following DEXA scan, the thoracic aorta was isolated and 2 adjacent segments $(\approx 4 \mathrm{~mm})$ were prepared. The PVAT was carefully cleaned from 1 segment and left intact on the other segment. Each aortic segment was placed on stainless steel hooks in phosphate-buffered solution. Aortas were equilibrated to $1 \mathrm{~g}$ tension, contracted 2 separate times with high potassium $\left(100 \mathrm{mM} \mathrm{K}^{+} ;+3\right.$ washes), phenylephrine challenged (PE; $0.1 \mathrm{nM}-30 \mu \mathrm{M}$ ), and exposed to acetylcholine (ACh; $0.1 \mathrm{nM}-30 \mu \mathrm{M})$. After 3 washes, aortas were contracted with thromboxane $\mathrm{A}_{2}$ analog (U46619) and relaxed with sodium nitroprusside (SNP; $0.1 \mathrm{nM}-10 \mu \mathrm{M})[24]$. 


\section{Scheme II: Aortic Section Removal}

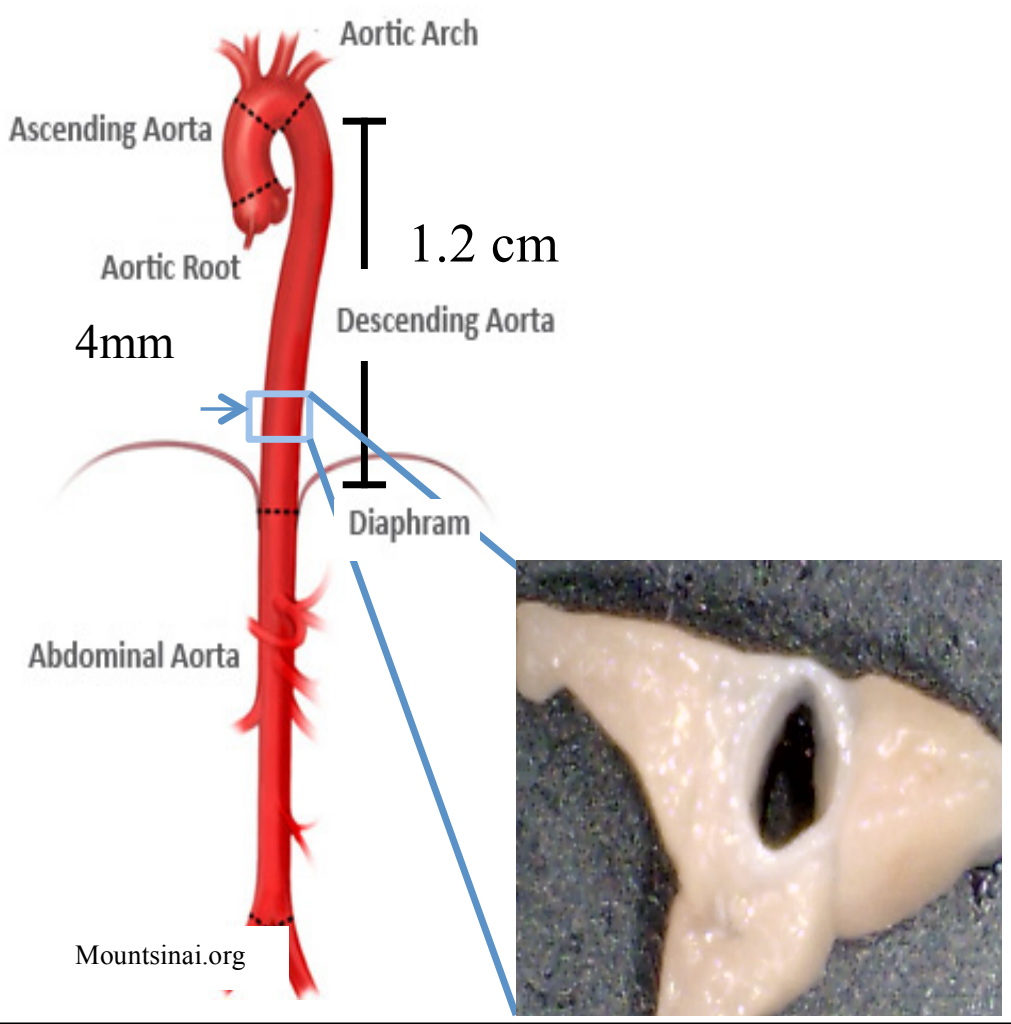

Scheme II: Illustration of aorta indicating location of distal thoracic aorta where segments were removed for study. Inset: Short segments (4mm length) were obtained from the distal thoracic aorta. Aortic sections were fixed in $10 \%$ neutral buffered formalin for aortic reactivity and PVAT morphometric analysis.

Histology: A $4 \mathrm{~mm}$ length of aorta was obtained from the distal thoracic aorta (Scheme

II). Aortic sections were placed in processing cassettes and fixed in $10 \%$ neutral buffered formalin (Leica Microsystems; Wetzlar, Germany). Histopathology was performed on formalin-fixed, paraffin-embedded aortic cross sections $(5 \mu \mathrm{M})$ stained with hematoxylin and eosin $(\mathrm{H} \& \mathrm{E})$ or fast green/Sirius red. Images were obtained at $\approx 100 \mathrm{x}$ magnification using a Spot advanced image capture software (SPOT Imaging Solutions, Sterling Heights, MI).

\section{PVAT Morphometry:}

Dorsal and Ventral Adipose Tissue: Perivascular adipose tissue (PVAT) is divided into ventral adipose tissue (VAT) and dorsal adipose tissue (DAT) sections (Scheme 3). All 
area measurements were obtained using IMAGEJ. These measurements were performed by outlining the outer edge of the VAT using IMAGEJ drawing tools, tracing the adventitia-VAT border, and completing a loop around the VAT. DAT measurements were similarly performed by outlining the outer edge of the DAT using IMAGEJ drawing tools, tracing the DAT-adventitia border, and completing the loop around the DAT. Scale calibration was performed using a scale calibrated to the image and magnification by spot software to obtain absolute measurements.

\section{Scheme III: Dorsal and Ventral Adipose Defined}

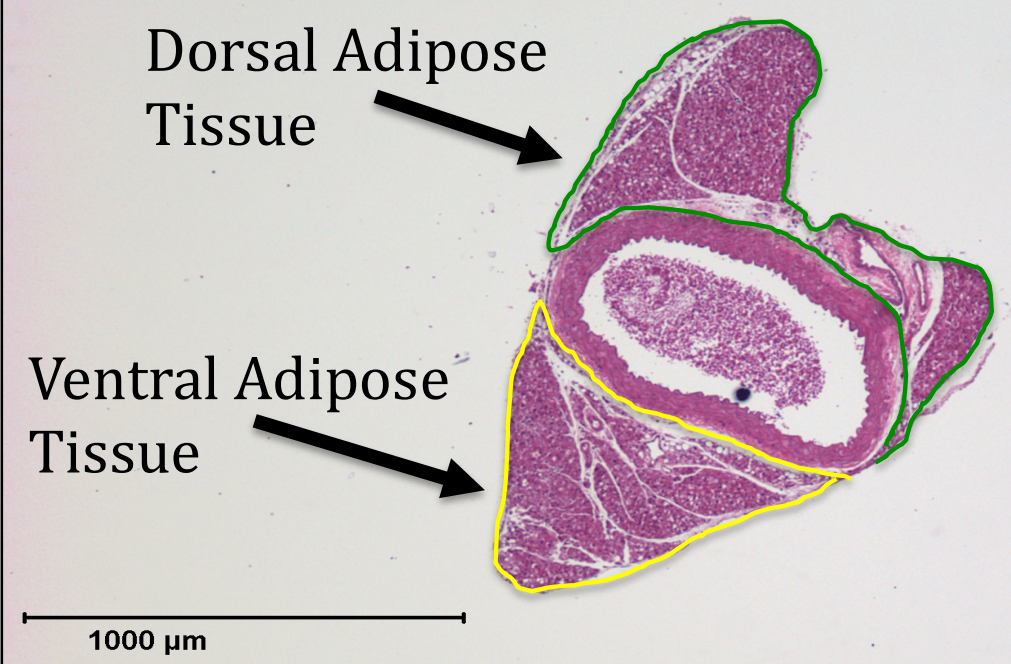

Scheme III: Low power micrograph of H\&E stained aortic cross section with defined areas of dorsal (DAT; green) and ventral adipose tissue (VAT; yellow) Mag. $=4 \mathrm{x}$.

Adipocyte Measurements: White adipocyte clusters were measured from the cross sections using NIH free software IMAGEJ (version 1.45s). Areas of clusters found in both the DAT and VAT were measured using IMAGEJ drawing tools. These clusters were defined as having $\geq 5$ adjacent white (unilocular) adipocytes (Scheme III). These 
clusters were typically found on the periphery of PVAT and usually surrounding blood vessels (vasa vasorum) near the aorta adventitia. The area of individual adipocytes present in these clusters was also measured using IMAGEJ drawing tools, and number of adipocytes in each cluster was manually counted.

\section{Figure 1: White Adipocyte Clusters}

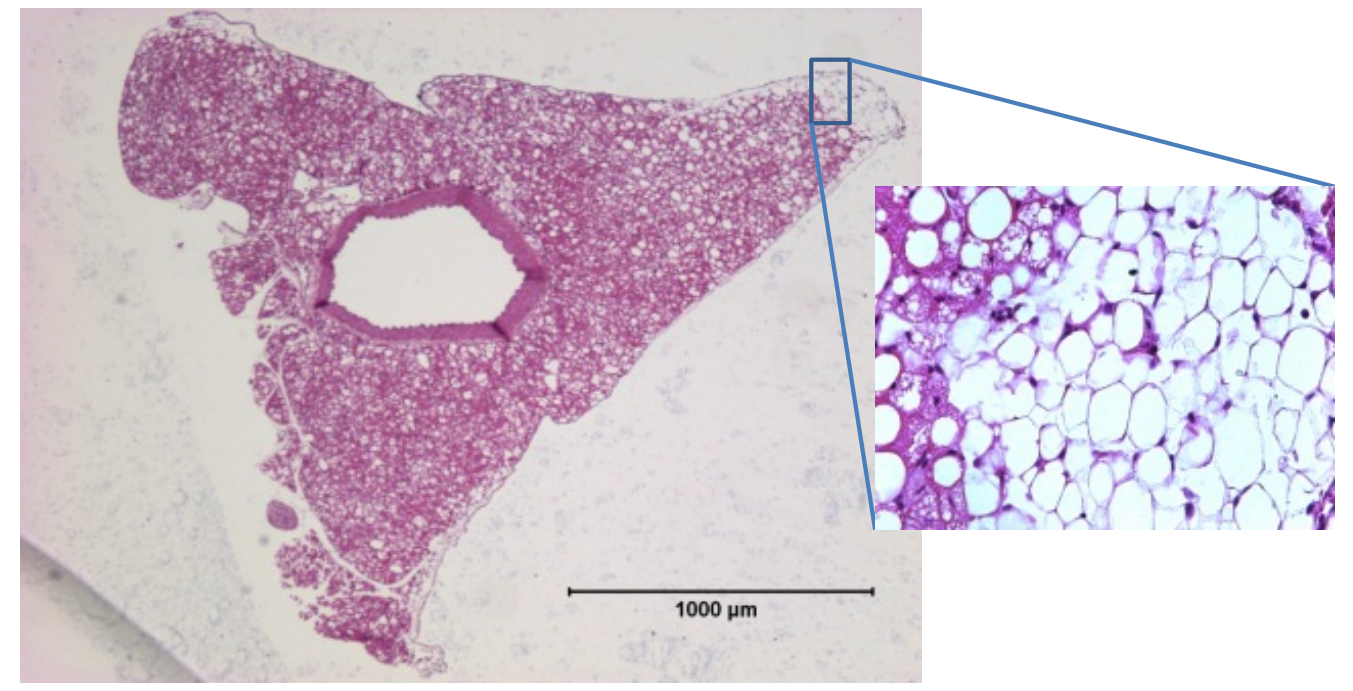

Figure 1: Low power micrograph of H\&E stained aortic cross section from a 22week-old AR-null mouse. Inset: Higher power image showing white adipocyte cluster present on the periphery of PVAT. Mag. $=4 \mathrm{x}$; inset: Mag. $=40 \mathrm{x}$

Statistics: Values are mean \pm S.E.M. Group data were compared using $t$ test, one-way analysis of variance with Holm-Sidak or Bonferroni post-test, or Kruskal-Wallis analysis of variance by ranks with Dunn's post test where appropriate (SigmaStat; SPSS, Inc., Chicago, IL). Significance level was set at $\mathrm{p}<0.05$.

\section{Results:}

DEXA Scan: High fat diet feeding significantly increased the percentage of fat measured using DEXA scan of both WT and AR-null mice (Fig.1). The largest gains were seen in the 22 -week old WT $(39.2 \% \pm 1.7 \%)$ and AR-null $(45.9 \% \pm 1.0 \%)$ mice, there was no 
difference in body fat percentage between NC 12-week and NC 22-week old animals in both groups (Fig. 1 A). When comparing 12-week old AR-null and WT mice on NC, ARnull mice had a significantly greater percentage fat than their WT counterparts (Fig. 1). A significant difference in fat percentage was also noted between 12-week old WT and ARnull mice fed HFD for 4 weeks. Additionally, a significant difference in body fat percentage was observed in comparisons between NC and HF 22-week old WT and ARnull mice.

PVAT Ring Analysis: Analysis of PVAT on fixed aortas revealed a significant increase in ring PVAT $(\mathrm{mg} / \mathrm{mm})$ in HF 12-week old AR-null mice when compared to the NC 12week old AR-null group (Fig.1 B). High fat diet also appeared to increase ring PVAT $(\mathrm{mg} / \mathrm{mm})$ in WT and AR-null 22-week old mice, but the increase was not significant. PVAT Area Measurements: PVAT area was measured using IMAGEJ. In WT mice, high fat feeding appeared to increase the area of DAT and total PVAT in both 12 and 22week old animals, however the data was not significant. In AR-null mice, DAT area appeared to increase with high fat feeding in both 12 week and 22-week-old mice. VAT area also increased with high fat diet, and there was a significant increase observed in HF 22-week old mice when compared to NC mice of the same age and genotype. When examining the entirety of the Perivascular adipose tissue (PVAT), area appeared to increase with high fat feeding, although the observed differences were not statistically significant.

White Adipocyte Cluster Analysis: In WT mice, HF feeding appeared to increase the cluster area in both the 12 and 22-week old groups. The greatest increase in cluster area was seen in the HF 22-week old group; however, none of these increases were determined to be significant (Fig.4 A). This could be attributed to the high variation of 
cluster area found in the HF animal groups. In AR-null mice, HF feeding appeared to increase cluster area as well, and cluster area in VAT also appeared to increase with age. Due to high variation in cluster size, there was no significant difference found between the groups. When comparing WT and AR-null groups, it appeared that AR-null mice had larger cluster areas initially and gained more cluster area than WT mice when fed a high fat diet. Particularly high variation was observed in the AR-null mice, which had the greatest cluster area gains under HF feeding found in their VAT. DAT cluster area appeared to decrease with age in AR-null mice, and HF diet had no effect to enlarge DAT clusters (Fig.4 B).

White Adipocyte Area Analysis: The area of individual white adipocytes in PVAT was measured using IMAGEJ. In WT mice, HF diet increased the area of white adipocytes in both the DAT and VAT. Of the WT mice, the largest increase in area was seen in the HF 22-week old group. DAT adipocytes in these mice had a significantly greater area than those of NC 22-week old WT mice. HF feeding also appeared to increase DAT adipocyte area in the HF 12-week old group, but a significant increase over NC 12-week old mice was not observed (Fig.4).

WT murine VAT adipocyte area was also increased by HF feeding. Twenty-twoweek old HF mice were seen to have significantly larger adipocytes in VAT than NC 22week old WT mice. Twelve-week-old HF fed mice also appeared to have larger adipocytes, although the data were not statistically significant. PVAT adipocytes altogether were increased in area by HF feeding. This increase was seen to be the greatest in the 22-week-old HF mice, whose adipocytes were significantly larger than those of NC 22-week-old WT mice (Fig.4). 
AR-null adipocyte size changes under HF diet were different than those seen in WT mice. HF feeding resulted in very little change in DAT adipocyte area of HF 12week old mice, and a slight increase in DAT adipocyte area in HF 22-week old mice. The greatest increase in adipocyte area under HF feeding was seen in VAT, particularly in 22week old HF mice. These mice showed significantly larger VAT adipocyte area compared to NC 22-week old AR-null mice. There appeared to be an increase in adipocyte area in VAT of HF 12-week old mice, but the data were not significant. No significant increase in DAT adipocyte area accompanied the VAT adipocyte area increase. Additionally, the mean area of adipocytes present in total PVAT was also significantly increased in 22-week old HF AR-null mice when compared to NC mice of the same age and genotype.

White Adipocyte Numbers: Total number of white adipocytes was enumerated from microscopy images. In WT mice, HF feeding appeared to have an effect to increase the number of adipocytes in both the 12 and 22-week old groups. Twelve-week old HF mice appeared to have an increase in mean VAT adipocyte number, as well as an increase in mean adipocyte number overall. Twenty-two week old HF mice had increased total adipocyte number overall, as well as in both DAT and VAT individually, however, these increases were not significant (Fig.5).

In AR-null mice, HF feeding appeared to have a different effect. Both 12- and 22week old HF mice appeared to have a decrease in mean adipocyte number in DAT, VAT, and overall. This difference appeared larger in the HF 12-week old group. However, the difference between the groups was not significant (Fig. 6).

When comparing diet and aged matched AR-null and WT mice, AR deficiency appeared to increase the number of cluster adipocytes in NC fed mice. Significantly more 
adipocytes were observed in VAT of 22-week old NC; AR-null mice when compared to WT mice of the same age and diet. Under HF conditions, AR-null mice had fewer cluster adipocytes than their WT counterparts, although no significant differences were observed.

Aorta Contractility Analysis: Aortas that were not cleaned of PVAT showed increased high potassium $(\mathrm{HI} \mathrm{K+)}$ contractility when compared to aortas that were cleaned of PVAT. Significant increases in contractility were seen in WT NC mice, as well as ARnull NC and HF mice (Fig.6A). In contrast, when aortas were contracted with a thromboxane $\mathrm{A}_{2}$ analog (U46619), PVAT presence significantly inhibited contractions in WT HF fed mice and both NC and HF AR-null mice (Fig. 6B). PVAT also exhibited an effect on acetylcholine (ACh) induced aortic relaxation (Fig. 6C). Intact PVAT on the WT HF fed group reversed endothelial dysfunction that was observed when the PVAT was removed from the WT HF aorta. In AR-null mice, ACh-induced relaxation was significantly enhanced in the HF fed group. Finally, sodium nitroprusside (SNP) relaxation appeared also to be enhanced by the presence of PVAT on aorta of NC and HF fed mice in both WT and AR-null groups (Fig. 6D)[24]. 
Figure 2: Total Body Fat and PVAT Ring Analysis

A

Body Fat Percentage

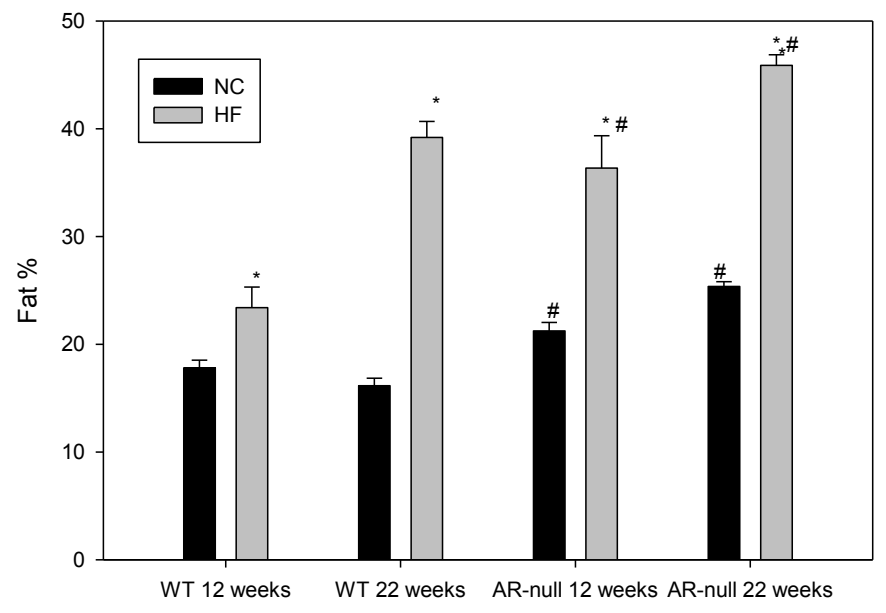

B PVAT Ring Analysis

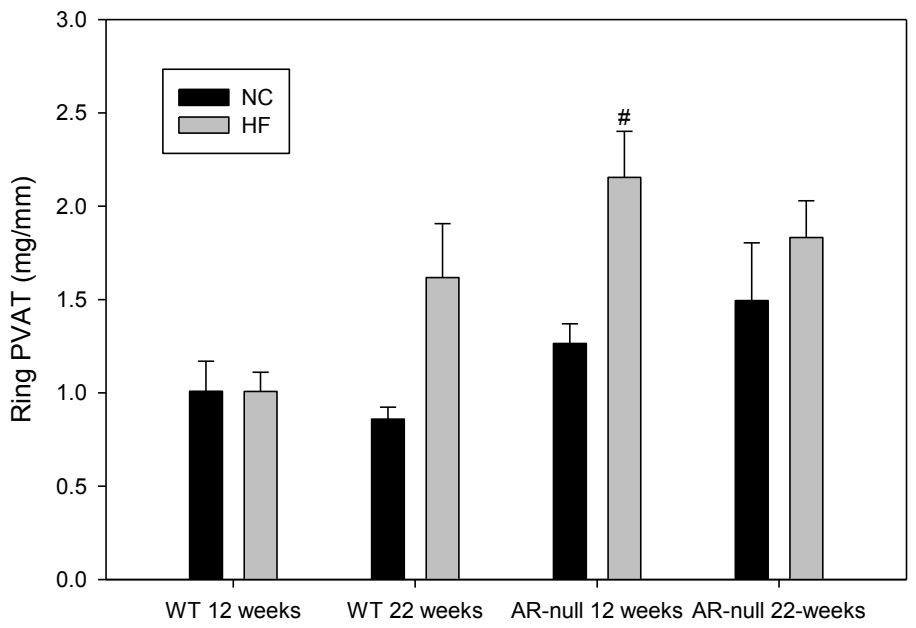

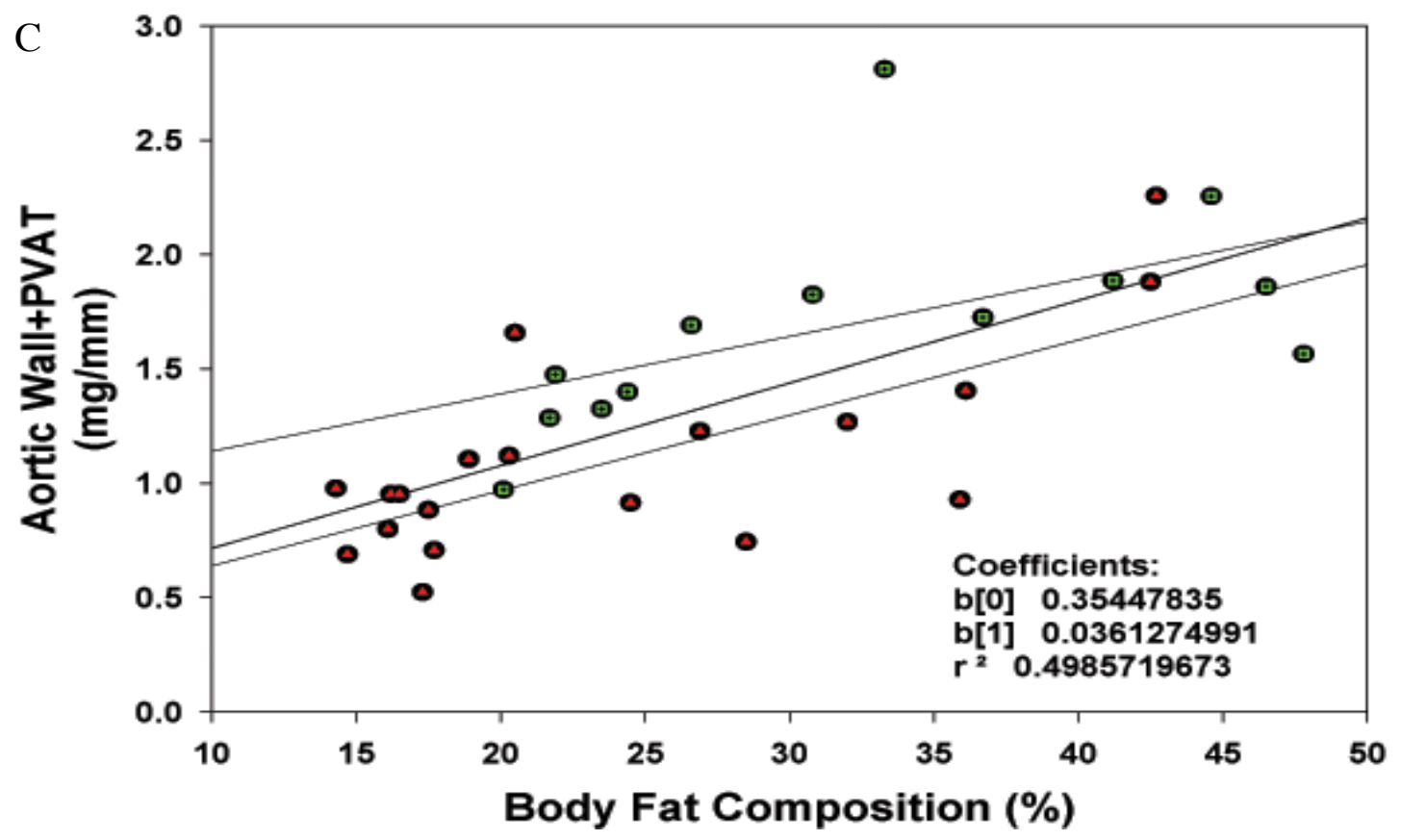

Figure 2: High fat diet increases the percent body fat of both AR-null and WT mice. Shown are graphs of mean percent body fat (A) of all groups, PVAT ring analysis (B), and a regression of PVAT vs. \% body fat $(\mathrm{C})$. Percent body fat was measured using DEXA-scan. * Indicates the data were significantly different than other within the group, and \# indicates the data is significantly different than the age and diet matched member of the other group $(\mathrm{p}<0.05)$. 
Figure 3: PVAT Area Analysis

A

WT PVAT Area

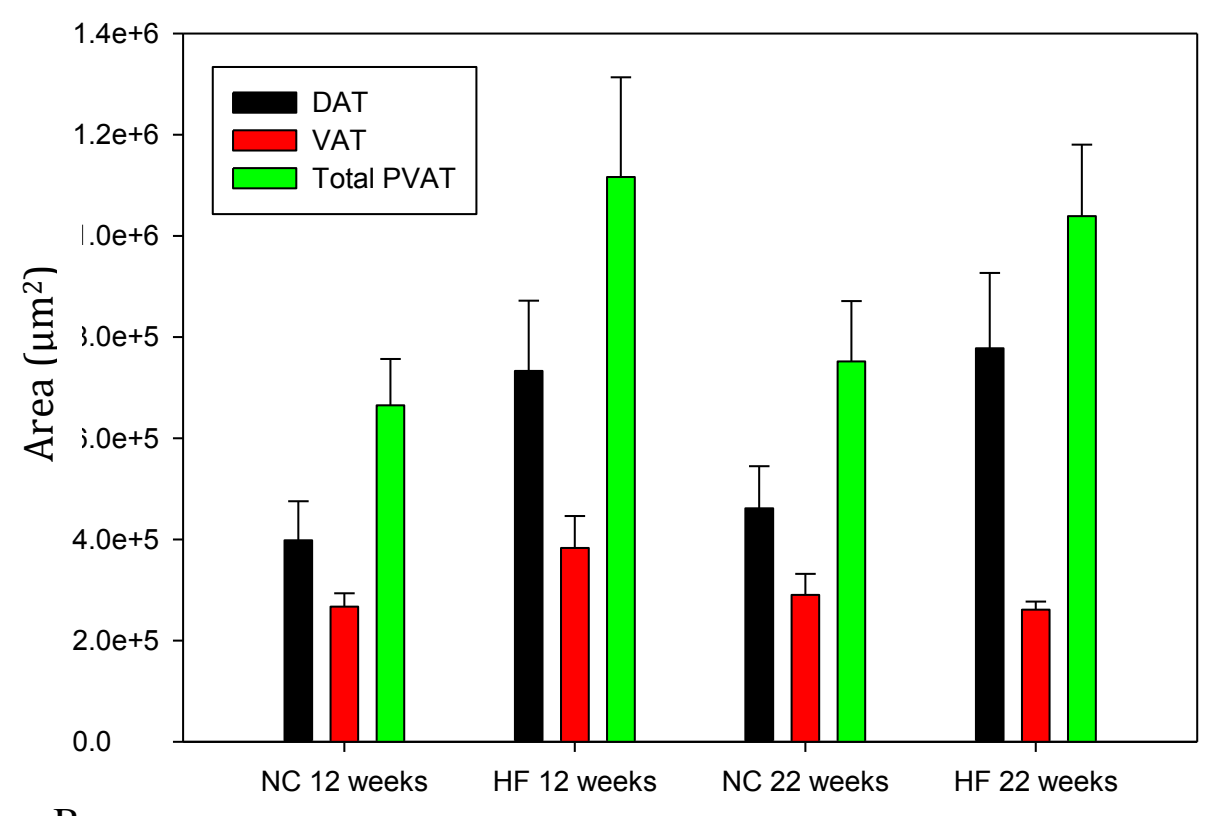

$\mathrm{B}$

AR-null PVAT Area

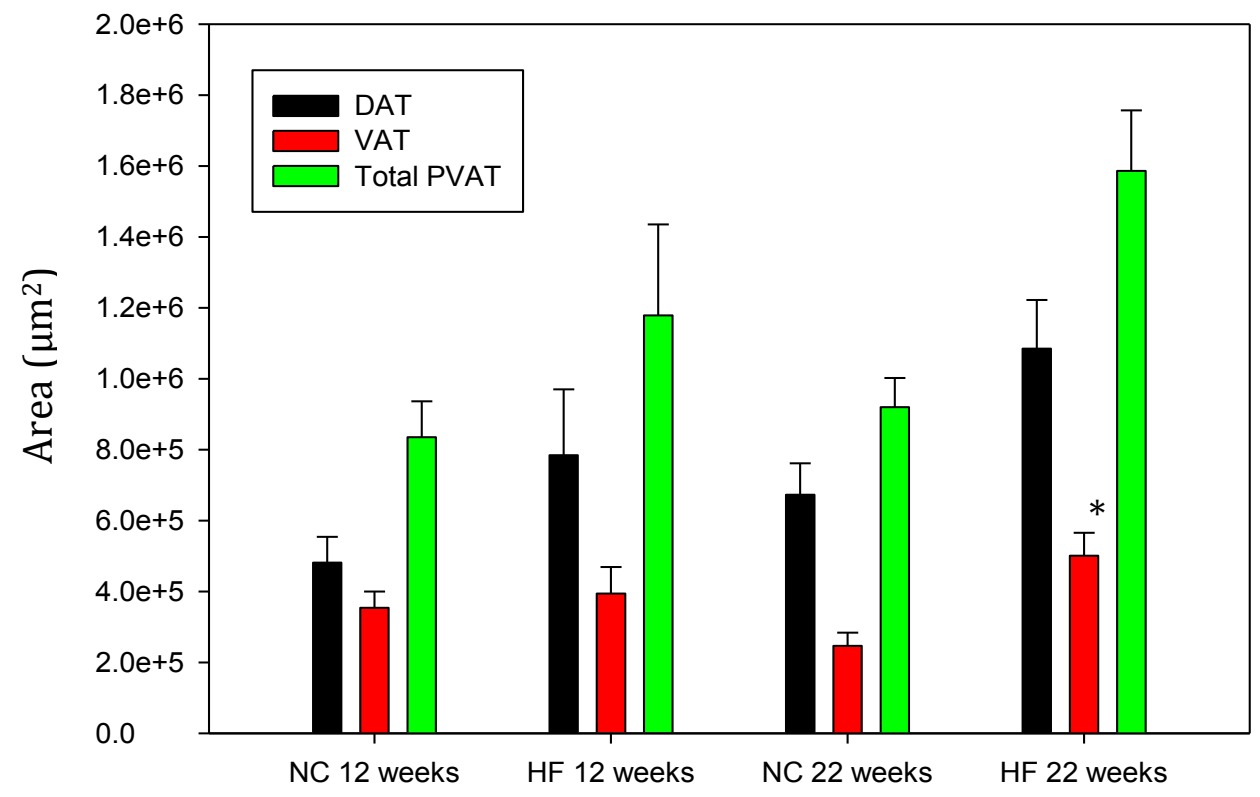

Figure 2: High fat diet affects size of PVAT. Graphs show WT (A) and AR-null (B) mean area $\left(\mu \mathrm{m}^{2}\right)$ of DAT, VAT, and total PVAT. * Indicates the data are significantly different than age and genotype matched mice $(\mathrm{p}<0.05)$. ARKO: NC 12-weeks $n=6$; HF 12-weeks $n=4$; NC 22-weeks $n=5$; HF 22-weeks $n=8$. WT: NC 12-weeks $n=5$; HF 12-weeks $n=7$; NC 22weeks $n=4$; HF 22-weeks $n=4$. 


\section{Figure 4: White Adipocyte Cluster Analysis \\ A \\ WT Cluster Area}
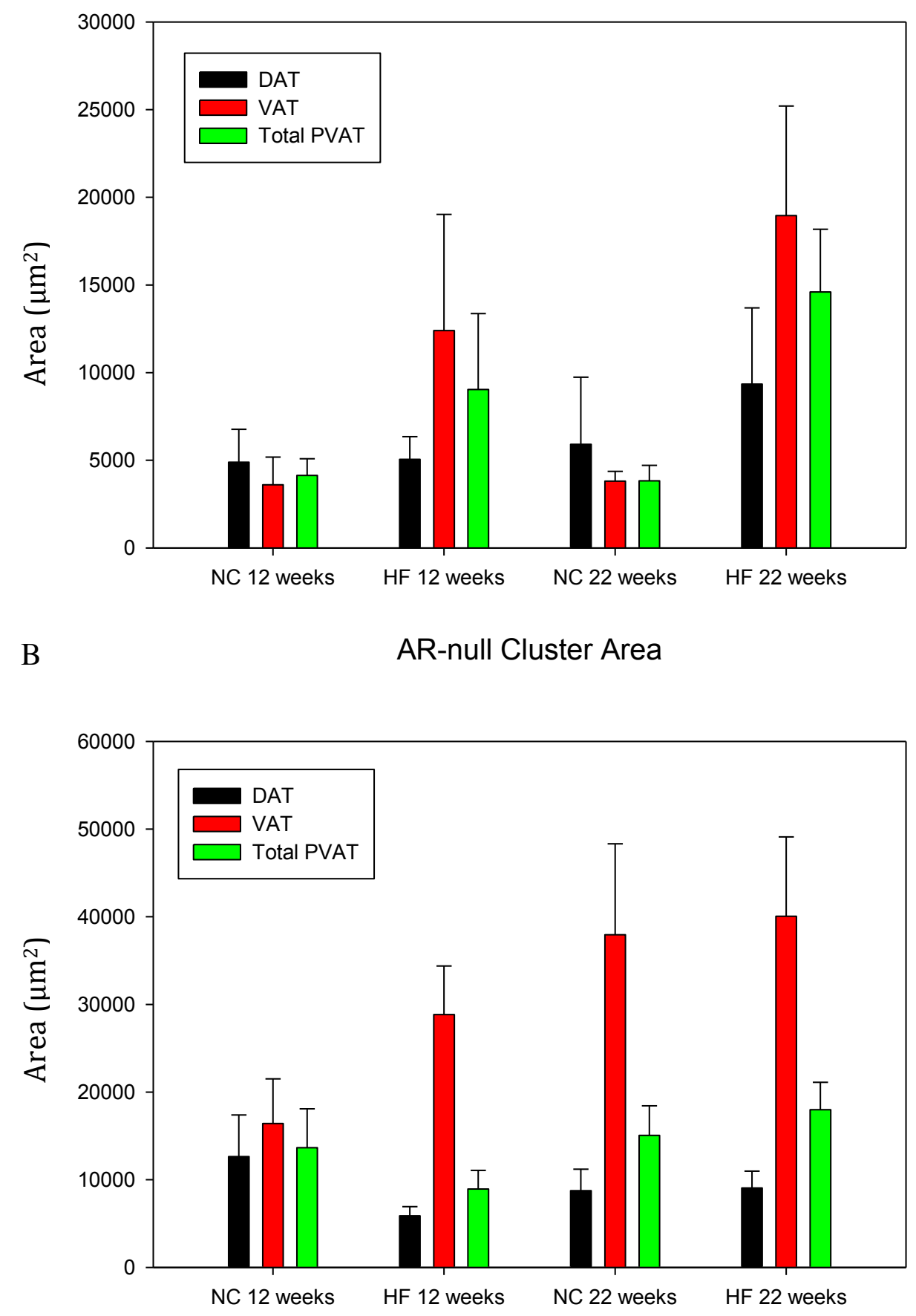

Figure 4: High fat diet effects on white adipocyte cluster area. Graphs show WT (A) and AR-null (B) mean area $(\mu \mathrm{m})$ of DAT, VAT, and total PVAT adipocyte clusters. ARKO: NC 12-weeks n=6; HF 12-weeks n=4; NC 22-weeks n=5; HF 22-weeks n=8. WT: NC 12-weeks $\mathrm{n}=5$; HF 12-weeks n=7; NC 22-weeks n=4; HF 22-weeks $\mathrm{n}=4$. 


\section{Figure 5: White Adipocyte Area Analysis}

\section{A \\ WT Adipocyte Area}
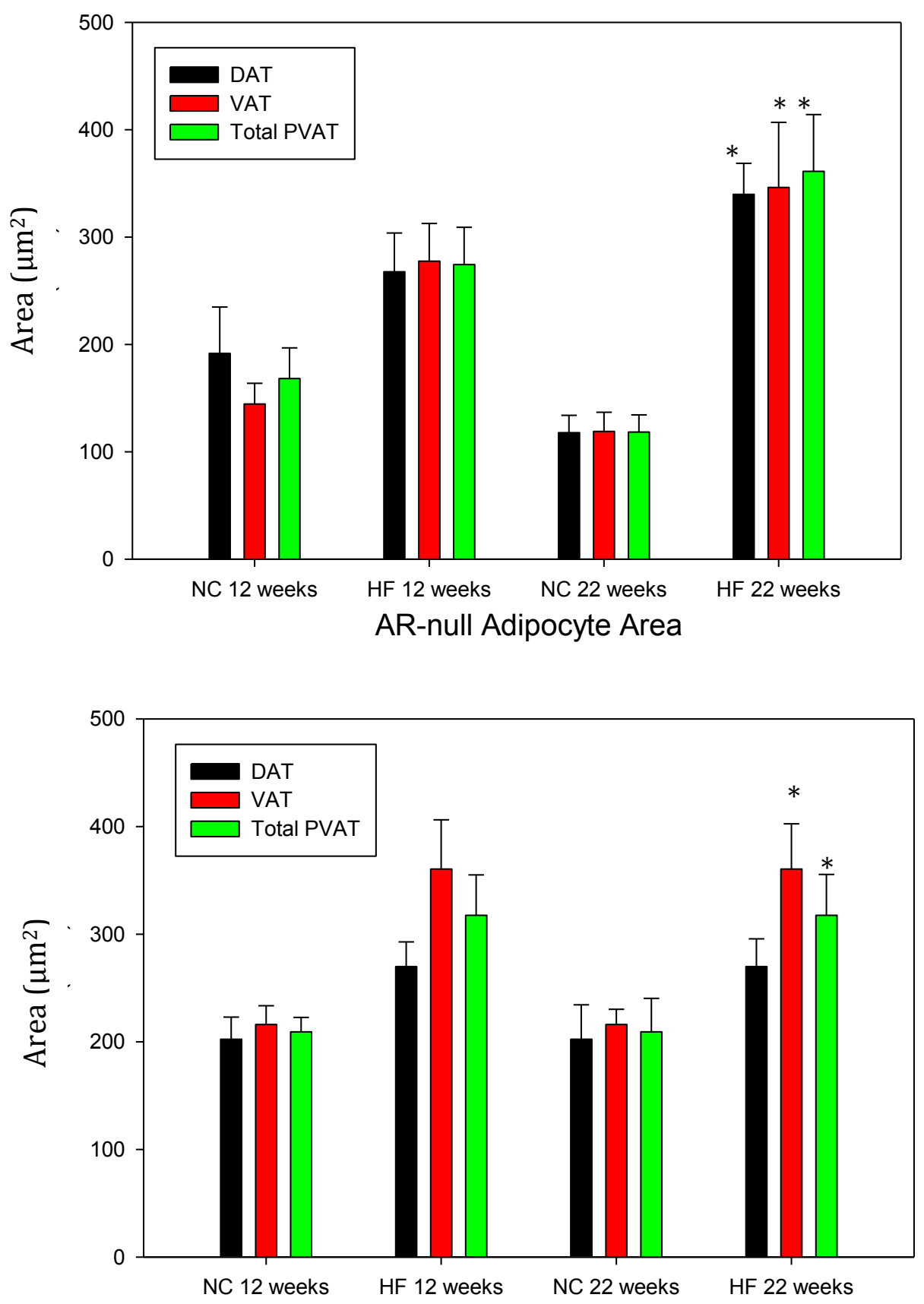

Figure 5: High fat diet effects on adipocyte area. Graphs show WT (A) and AR-null (B) mean area $\left(\mu \mathrm{m}^{2}\right)$ of DAT adipocytes, VAT adipocytes, and total PVAT adipocytes. * Indicates the data are significantly different than genotype and age matched mice of differing diets $(\mathrm{p}<0.05)$. ARKO: $\mathrm{NC} 12$-weeks $\mathrm{n}=6$; HF 12-weeks $\mathrm{n}=4$; NC 22-weeks $\mathrm{n}=5$; HF 22-weeks n=8. WT: NC 12-weeks n=5; HF 12-weeks n=7; NC 22-weeks n=4; HF 22weeks $\mathrm{n}=4$. 


\section{Figure 6: Cluster Adipocyte Enumeration}

A

WT Cluster Adipocyte Number

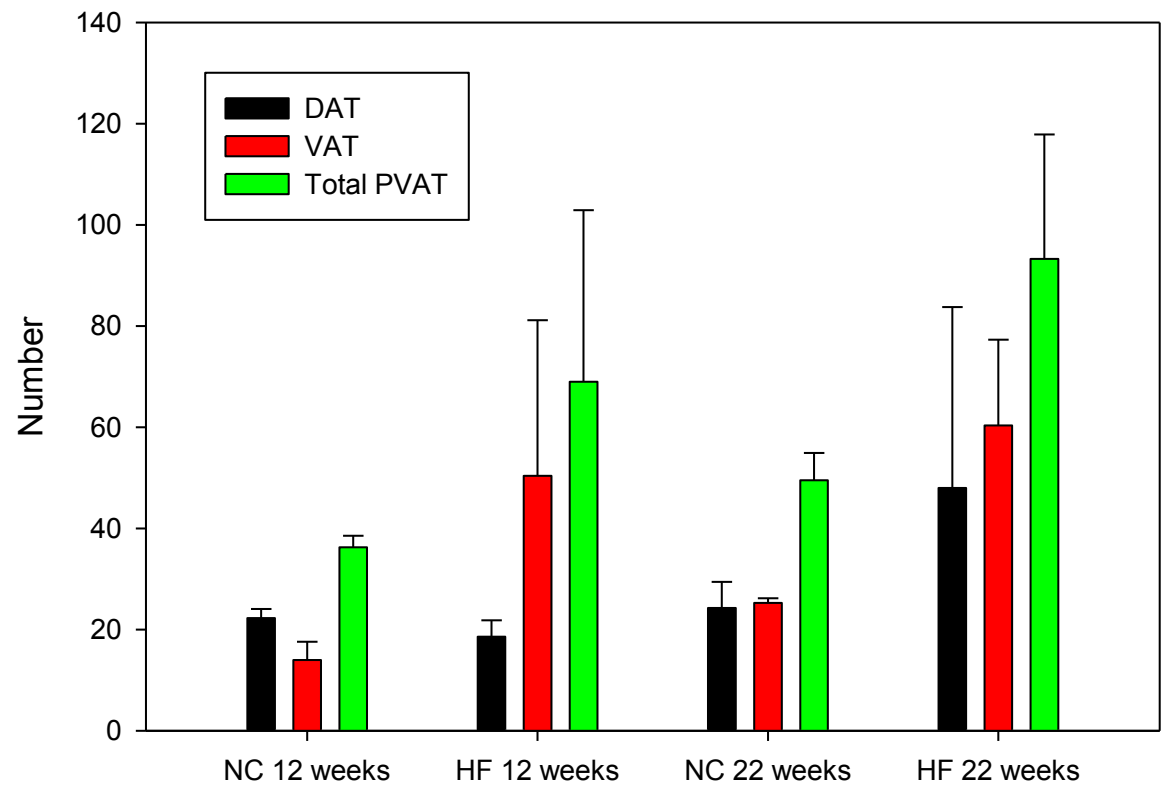

B

AR-null Cluster Adipocyte Number

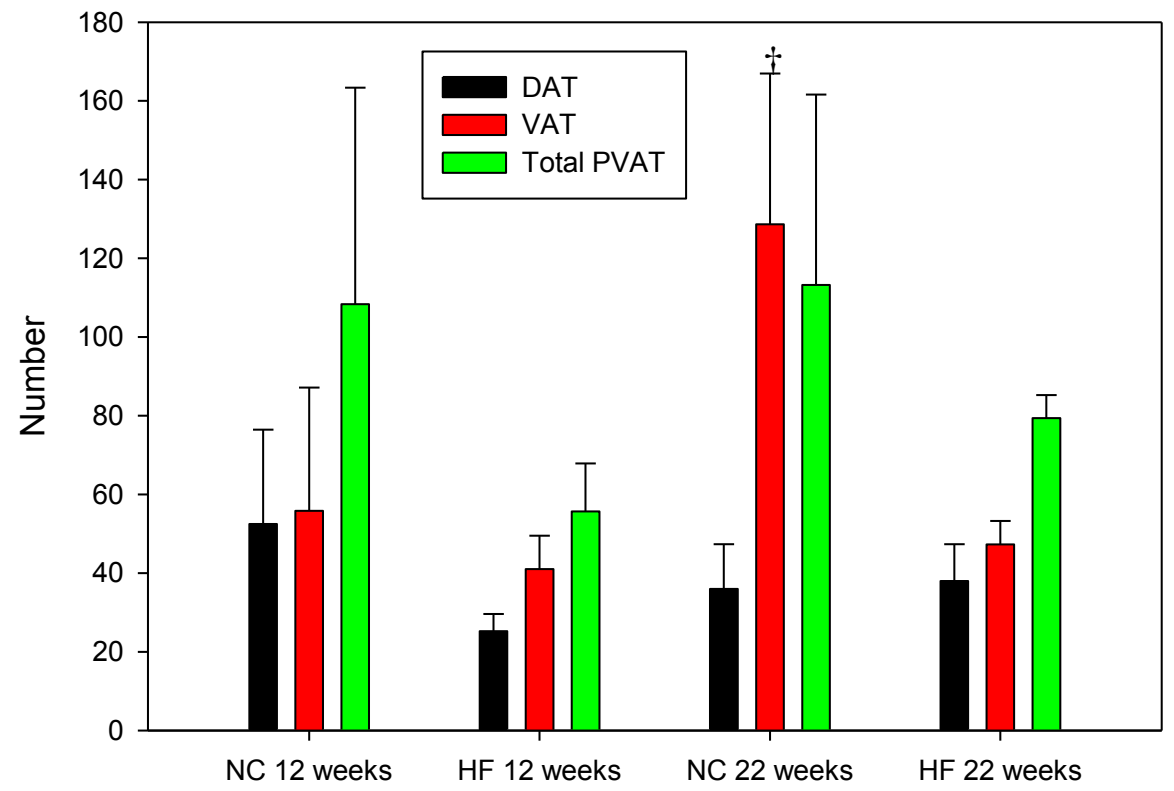

Figure 6: High fat diet effects on adipocyte number. Graphs show WT and AR-null mean number of adipocytes in DAT, VAT, and total PVAT slides. $\dagger$ Indicates the data are significantly greater than diet and age matched mice of differing genotypes $(p<0.05)$. ARKO: NC 12-weeks n=6; HF 12-weeks n=4; NC 22-weeks n=5; HF 22-weeks $n=8$. WT: NC 12-weeks n=5; HF 12-weeks n=7; NC 22-weeks n=4; HF 22-weeks n=4. 


\section{Figure 7: Aortic Reactivity Analysis}

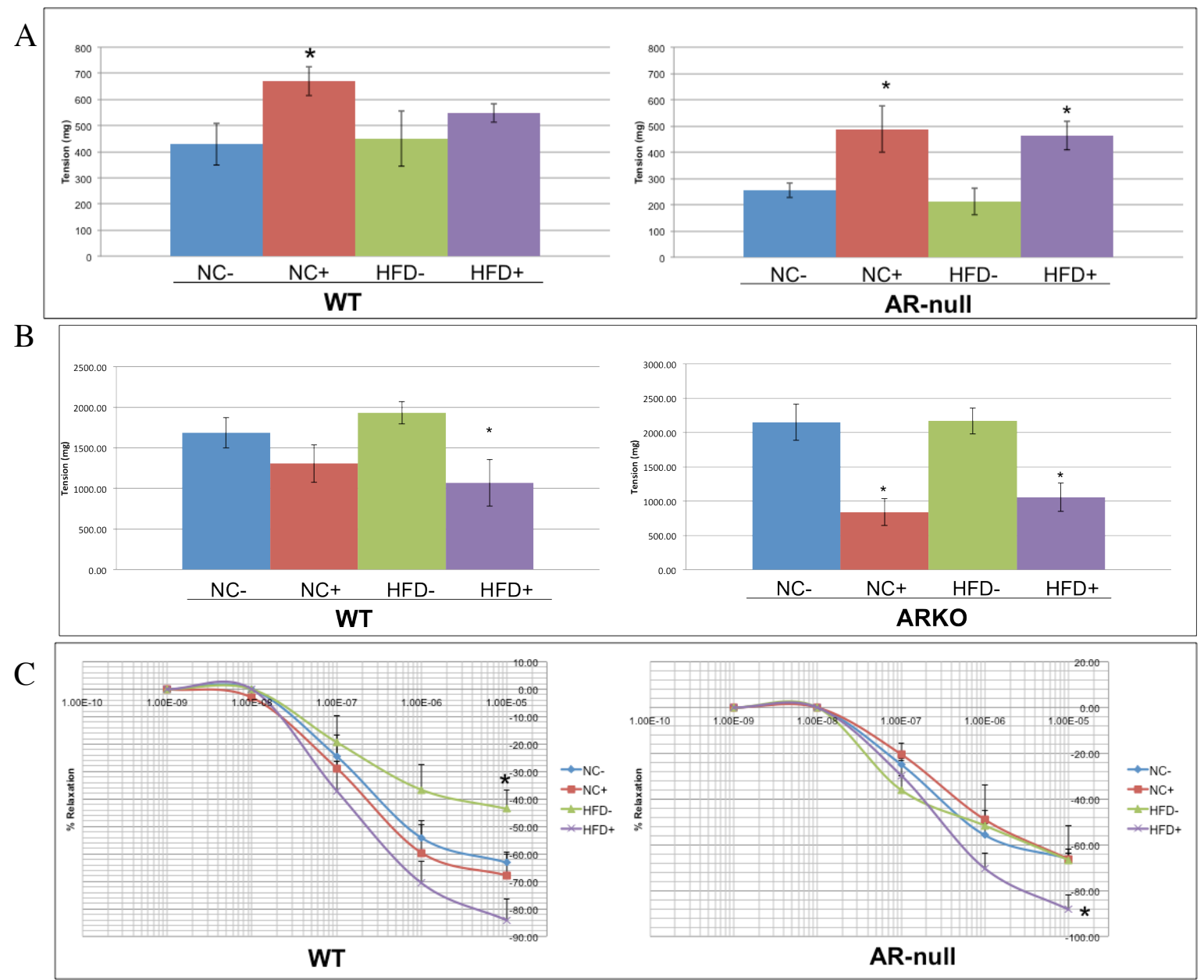

$\mathrm{D}$
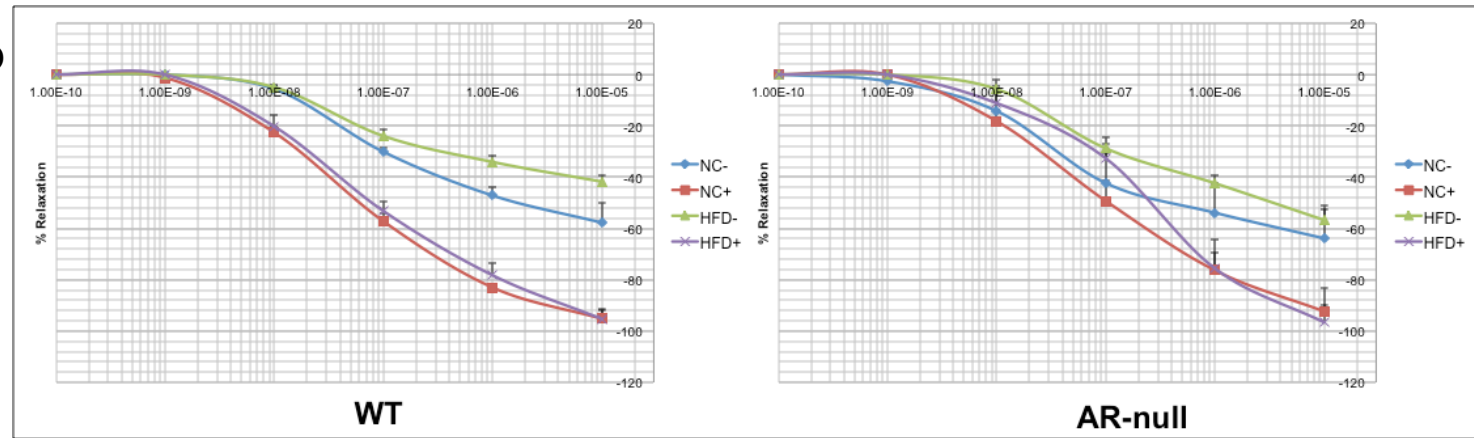

Figure 6: High potassium-induced contractions in WT and ARKO mice (A). PVAT increased $\mathrm{HI} \mathrm{K}+$ contractility is increased in aortas with PVAT intact $(\mathrm{NC}+, \mathrm{HFD}+)$ compared to aortas devoid of PVAT (NC-, HFD-). PVAT is enhancing contractility in response to high potassium (A). Thromboxane $\mathrm{A}_{2}$ agonist contractions in WT and ARKO mice (B). In both groups, presence of PVAT $(+)$ significantly inhibited U46619-induced contraction (B). Acetylcholineinduced aortic relaxation in WT and ARKO mice $(C)$. Where PVAT was intact in the WT HFD-fed group (HFD+), endothelial dysfunction (HFD-) was reversed. In ARKO mice, PVAT in HFD group (HFD+) significantly enhanced ACh-induced relaxation over HFD- (C). SNPinduced aortic relaxation in WT and ARKO mice (D). PVAT enhanced SNP-induced relaxation (as \%) in both NC and HFD fed WT and ARKO mice (D). N=4 for all groups 


\section{Discussion:}

This study demonstrates for the first time that short-term high fat feeding results Under high fat feeding conditions, body fat percentage significantly increased in all groups, as expected. AR-null mice had a higher percent body fat that their diet and age matched WT counterpart. It is of interesting note that while AR-null mice had an accelerated obesity level of WT mice, they did not exhibit endothelial dysfunction, as was observed in WT HF fed mice. Additionally, PVAT mass also appeared to increase with HF feeding, with a greater increase in mass occurring in AR-null mice. These results indicate that increases in PVAT adiposity mirror overall body adiposity.

In addition to an increase in the mass of PVAT, area was also increased under high fat feeding conditions. The greatest gains in area appeared to be in DAT, although a relatively high variance led to a lack of statistical significance for these measurements. AR-null mice appeared to have a greater PVAT area when compared to WT age and diet matched counterparts, suggesting that AR plays a role in proliferation/growth of adipose depots. DAT is more vascularized than VAT, and because this is where the greatest amount of growth occurs a likely explanation for this growth would be the infiltration of adipose stem cells, likely to be unique to PVAT [7, 25].

The area of white adipocyte clusters was highly variable in this study. In ARnull mice, HF feeding appeared to increase cluster area in VAT, while cluster area in DAT remained the same. A similar effect was seen as AR-null mice aged, independent of diet. While these trends in AR-null mice were not significant, further study may find that VAT white adipocyte clusters are larger than others. White adipocyte cluster areas of WT mice were also highly variable through this study, but weak trends can be noticed from the data. Overall, HF diet appeared to increase the area of white adipocyte clusters 
throughout the PVAT. It appeared that a majority of these clusters occur on the periphery of PVAT or adjacent to a blood vessel. From these observations, it is possible that white PVAT adipocyte stem cells are entering through the bloodstream or that the primarily brown multilocular adipose of PVAT differentiates to a white unilocular phenotype under high fat conditions.

Overall white adipocyte size appeared to increase with high fat feeding. In WT mice area increase was uniform across the entirety of PVAT with both DAT and VAT white adipocytes increasing similarly in area. AR-null mice showed a greater increase in adipocyte area in the VAT region of PVAT than in DAT. Mice aged 22 weeks showed larger gains in adipocyte size under HF conditions than 12 week-old, suggesting that age plays a role in the size increase of these cells. Additionally, AR may also be important in the localization/ growth of these larger adipocytes.

When examining the numbers of white adipocytes present in clusters, WT mice increased the number of white adipocytes present in clusters. Interestingly, it appears that HF fed mice of AR-null groups have a lower number of cluster adipocytes than NC mice. This lower number might be important in the compensatory effects of PVAT under HF conditions. Studies of white unilocular PVAT show that it expresses high levels of inflammatory cytokines [6] and dispersal of these white adipocytes throughout the brown PVAT may diminish the ability to elicit an inflammatory response. Due to the high variability observed in enumeration, however, these results should be cautiously interpreted.

Following high fat feeding, aortas from WT mice aged 22 weeks exhibited endothelial dysfunction that was detected as a decreased relaxation response to ACh. When PVAT was left on the aorta, endothelial dysfunction was no longer detected. These 
results support the idea that PVAT plays an important role in vascular function. Additionally, they suggest that perivascular adipose may be playing a part in the maintenance of normal function under adverse physiological conditions.

Functional analysis of both WT and AR-null aortas revealed that the presence of PVAT modifies aortic response to several different vasoactive compounds in a similar way. Aortas with PVAT present showed a weaker contraction when exposed to a thromboxane $\mathrm{A}_{2}$ agonist. This response to the thromboxane agonist likely demonstrates the action of adipose derived relaxing factors. These ARDFs are proposed to act on voltage dependent $\mathrm{K}+$ channels in the membrane of vascular smooth muscle cells, resulting in hyperpolarization [26]. This idea is further supported by the increased high potassium contractility of PVAT positive aortas. The increase in external $\mathrm{K}+$ levels result in a reduced difference between $\mathrm{K}+$ equilibrium potential and the electric potential of the membrane, which diminishes any effect a K+ channel opener would exhibit [9]. Perivascular adipose presence appears to enhance the relaxation of mouse aortas under high fat feeding conditions. In this study, HF fed WT mouse aortas developed endothelial dysfunction, which presented as a diminished ACh relaxation. When PVAT was left on these aortas, endothelial dysfunction was reversed. These results suggest that PVAT is exhibiting a compensatory effect on the aorta under adverse physiological conditions, in this case high fat feeding and obesity. This could be a result of an increased action/expression of ADRFs, but further studies would need to be conducted to test this explanation. When examining SNP-induced relaxation, PVAT positive aortas from both NC and HF animals appeared to have responded with a greater amount of relaxation, although there was no significant difference in the data. Again, this is potentially the result of ADRF action on the aorta. 
There are several limitations that should be noted in this study. First, a small number of animals were used for each group and because of this, our results may not accurately reflect what occurs in the population under these experimental conditions. Additionally, only male mice were used in this study, but this is due to the fact that males are more susceptible to cardiovascular disease than females. While most current PVAT research explores its detrimental properties, this study demonstrates that PVAT in an important tissue that is still very much a mystery. Studies of white PVAT have revealed it to be inflammatory in nature and its size is correlated to cardiovascular disease risk [6, 13]. Conversely, in our present study we focused on the brown adipose of PVAT and functional data reveal that offers compensatory benefits under short-term high fat diet stress. Under long-term high fat diet, PVAT may eventually lose its compensatory benefits and become increasingly inflammatory, reducing its ability to release ARDFs and raising the risk for cardiovascular problems via the release of contractile factors. 


\section{Acknowledgements:}

I would like to express my gratitude to Dr. Daniel J. Conklin for his mentorship and guidance over the course of this study. Without him, this project would have never come to fruition. Additionally, I also thank Dr. Petra Haberzettl for familiarizing me with many of the materials and techniques necessary for the completion of this study. I would also like to thank Emily Steinmetz for her assistance and Ken MacKinlay for performing the aorta reactivity studies. Finally, I would like to thank the thesis committee: Dr. Daniel J. Conklin, Dr. Cynthia Corbitt, and Dr. Joseph Steffen, for their time in reviewing this thesis. Grant support provided by: SROP 2011 (Dr. P. Feldhoff, Director), NIGMSfunded Diabetes and Obesity Center (Dr. A. Bhatnagar, Director; RR024489), and HL89380 (DJC). 


\section{References:}

1. Srivastava, S., et al., Structural and kinetic determinants of aldehyde reduction by aldose reductase. Biochemistry, 1999. 38(1): p. 42-54.

2. $\quad$ Flegal, K.M., et al., Prevalence and Trends in Obesity Among US Adults, 19992008. Jama-Journal of the American Medical Association, 2010. 303(3): p. 235241.

3. Houle, B., et al., Use of density-equalizing cartograms to visualize trends and disparities in state-specific prevalence of obesity: 1996-2006. Am J Public Health, 2009. 99(2): p. 308-12.

4. Sironi, A.M., et al., Impact of increased visceral and cardiac fat on cardiometabolic risk and disease. Diabet Med, 2011.

5. Goldstein, B.J., Insulin resistance as the core defect in type 2 diabetes mellitus. Am J Cardiol, 2002. 90(5A): p. 3G-10G.

6. Chatterjee, T.K., et al., Proinflammatory phenotype of perivascular adipocytes: influence of high-fat feeding. Circ Res, 2009. 104(4): p. 541-9.

7. Rajsheker, S., et al., Crosstalk between perivascular adipose tissue and blood vessels. Curr Opin Pharmacol, 2010. 10(2): p. 191-6.

8. Gollasch, M., Vasodilator signals from perivascular adipose tissue. $\mathrm{Br} \mathrm{J}$ Pharmacol, 2011.

9. Lohn, M., et al., Periadventitial fat releases a vascular relaxing factor. FASEB J, 2002. 16(9): p. 1057-63.

10. Rebolledo, A., et al., Early alterations in vascular contractility associated to changes in fatty acid composition and oxidative stress markers in perivascular adipose tissue. Cardiovasc Diabetol, 2010.9(1): p. 65.

11. $\mathrm{Gu}, \mathrm{P}$. and $\mathrm{A}$. Xu, Interplay between adipose tissue and blood vessels in obesity and vascular dysfunction. Rev Endocr Metab Disord, 2013. 14(1): p. 49-58.

12. Takaoka, M., et al., Periadventitial adipose tissue plays a critical role in vascular remodeling. Circ Res, 2009. 105(9): p. 906-11.

13. Verhagen, S.N. and F.L. Visseren, Perivascular adipose tissue as a cause of atherosclerosis. Atherosclerosis, 2011.214(1): p. 3-10.

14. Manka, D., et al., Abstract 15733: Perivascular Adipose Tissue Triggers Vasa Vasorum Formation and Injury-Induced Neointimal Hyperplasia in Mice. Circulation, 2010. 122(A15733).

15. Guzik, T.J., et al., Perivascular adipose tissue as a messenger of the brain-vessel axis: role in vascular inflammation and dysfunction. J Physiol Pharmacol, 2007. 58(4): p. 591-610.

16. Tomlinson, D.R., G.B. Willars, and A.L. Carrington, Aldose reductase inhibitors and diabetic complications. Pharmacol Ther, 1992. 54(2): p. 151-94.

17. van Gerven, J.M. and A.T.A.M. Tjon, The efficacy of aldose reductase inhibitors in the management of diabetic complications. Comparison with intensive insulin treatment and pancreatic transplantation. Drugs Aging, 1995. 6(1): p. 9-28.

18. Srivastava, S.K., K.V. Ramana, and A. Bhatnagar, Role of aldose reductase and oxidative damage in diabetes and the consequent potential for therapeutic options. Endocr Rev, 2005. 26(3): p. 380-92. 
19. Raitakari, M., et al., Weight reduction with very-low-caloric diet and endothelial function in overweight adults: role of plasma glucose. Arterioscler Thromb Vasc Biol, 2004. 24(1): p. 124-8.

20. Blomkalns, A.L., T. Chatterjee, and N.L. Weintraub, Turning ACS outside in: linking perivascular adipose tissue to acute coronary syndromes. Am J Physiol Heart Circ Physiol, 2010. 298(3): p. H734-5.

21. Fitzgibbons, T.P., et al., Similarity of mouse perivascular and brown adipose tissues and their resistance to diet-induced inflammation. Am J Physiol Heart Circ Physiol, 2011. 301(4): p. H1425-37.

22. Wang, H., et al., Obesity-induced endothelial dysfunction is prevented by deficiency of P-selectin glycoprotein ligand-1. Diabetes, 2012. 61(12): p. 321927.

23. Ho, H.T., et al., Aldose reductase-deficient mice develop nephrogenic diabetes insipidus. Mol Cell Biol, 2000. 20(16): p. 5840-6.

24. Conklin, D.J., et al., Abstract 19716: Role of Aldose Reductase in Perivascular Adipose Tissue and Vascular Dysfunction in Short-Term Diet-Induced Obesity Circulation, 2012. 126(A19716).

25. Tchkonia, T., et al., Identification of depot-specific human fat cell progenitors through distinct expression profiles and developmental gene patterns. Am J Physiol Endocrinol Metab, 2007. 292(1): p. E298-307.

26. Gollasch, M., Vasodilator signals from perivascular adipose tissue. Br J Pharmacol, 2012. 165(3): p. 633-42. 